\title{
On the Lagrangian and Hamiltonian description of the damped linear harmonic oscillator
}

\author{
V. K. Chandrasekar, M. Senthilvelan, M. Lakshmanan \\ Centre for Nonlinear Dynamics, Department of Physics, Bharathidasan \\ University, Tiruchirappalli - 620 024, India
}

\begin{abstract}
Using the modified Prelle- Singer approach, we point out that explicit time independent first integrals can be identified for the damped linear harmonic oscillator in different parameter regimes. Using these constants of motion, an appropriate Lagrangian and Hamiltonian formalism is developed and the resultant canonical equations are shown to lead to the standard dynamical description. Suitable canonical transformations to standard Hamiltonian forms are also obtained. It is also shown that a possible quantum mechanical description can be developed either in the coordinate or momentum representations using the Hamiltonian forms.
\end{abstract}

Key words: Lagrangian and Hamiltonian approach, Lagrangian and Hamiltonian mechanics, Canonical formalism, Lagrangians and variational principles, Quantum mechanics.

PACS: 11.10.Ef, 45.20.Jj, 04.20.Fy, 03.65.-w

\section{Introduction}

In a recent paper $[1,2]$, we have studied the dynamical aspects of the Liénard type nonlinear oscillator equation

$$
\ddot{x}+k x \dot{x}+\frac{k^{2}}{9} x^{3}+\lambda x=0
$$

where over dot denotes differentiation with respect to $t$ and $k$ and $\lambda$ are arbitrary parameters, and shown that the frequency of oscillations of a nonlinear oscillator need not depend upon the amplitude [1]. Our studies have also revealed that the system (1), eventhough deceptively appears to be a dissipative 
one, admits conservative Hamiltonian description for all values of the parameters $k$ and $\lambda$. In fact, the conventional criterion that the divergence of the flow function vanishes for a Hamiltonian system fails here in the original variables. These facts motivate one to investigate further the possible existence of time independent Hamiltonian functions in actual dissipative systems.

For our present study, we consider the ubiquitous damped linear harmonic oscillator

$$
\ddot{x}=-(\alpha \dot{x}+\lambda x) \equiv \phi(x, \dot{x})
$$

where $\alpha$ and $\lambda$ are the damping parameter and strength of the forcing, respectively. When damping is absent, $\alpha=0$, the frequency of oscillation $\omega=\sqrt{\lambda}>0$. For $\lambda<0$, one has unbounded motion. System (2) is known to possess a time-dependent Lagrangian [3-5, 7, 15], $L=e^{\alpha t}\left(\frac{1}{2} \dot{x}^{2}-\frac{\lambda}{2} x^{2}\right)$, and a Hamiltonian $H=\frac{1}{2} p^{2} e^{-\alpha t}+\frac{\lambda}{2} x^{2} e^{\alpha t}$. For the past several decades there has been a number of attempts to quantize the damped linear harmonic oscillator (see for example, Refs. [6-20]) from different points of view, but it appears that the problem still eludes a completely satisfactory resolution. One of the major conceptual difficulties seem to be the lack of an appropriate time independent Hamiltonian formalism. In this paper, we report such a formalism by making use of the recently developed modified Prelle-Singer approach to identify integrals of motion and integrability of dynamical systems [21].

System (2) is obviously a dissipative one for $\alpha>0$ (and growing one for $\alpha<0)$, since $\frac{d}{d t}\left(\frac{1}{2} \dot{x}^{2}+\frac{1}{2} \lambda x^{2}\right)=\frac{d E}{d t}=-\alpha \dot{x}^{2}<0$, where $E=\left(\frac{1}{2} \dot{x}^{2}+\frac{1}{2} \lambda x^{2}\right)$ is the total energy of the undamped oscillator. From a dynamical point of view Eq. (2) can be written as a system of coupled first order ordinary differential equations (ODEs)

$$
\begin{aligned}
& \dot{x}=y \\
& \dot{y}=-\lambda x-\alpha y
\end{aligned}
$$

so that the divergence of the flow function

$$
\Lambda=\frac{\partial \dot{x}}{\partial x}+\frac{\partial \dot{y}}{\partial y}=-\alpha<0,
$$

which is the standard criterion for dissipative systems. Yet, we show here that system (2) possesses a time independent integral of motion for all values of the parameters $\lambda$ and $\alpha$. We also provide the explicit form of the Lagrangian and Hamiltonian associated with Eq. (2) in different parametric regimes and derive all the expected solutions from the resulting canonical equations of motion. Our results also show that the dissipative dynamical systems can 
also have conservative Hamiltonian description, which can aid in appropriate quantization procedure as will be indicated.

The plan of the paper is as follows. In Section 2, we point out how the time independent integrals can be obtained for the damped linear harmonic oscillator (2). Then in Section 3, we identify an appropriate time-independent Lagrangian and so a time-independent Hamiltonian in the appropriate parameter ranges. In Section 4, we show that through the resulting canonical equations of motion, we obtain the correct explicit solutions. In Section 5, we succeed to identify suitable canonical transformations to identify 'standard' Hamiltonian description. Finally, in Section 6, we discuss briefly the possible quantum formulation of the problem. Section 7 summarizes our results.

\section{Extended Prelle-Singer procedure}

To begin with we assume that the ODE (2) admits a first integral $I(t, x, \dot{x})=$ $C$, with $C$ constant on the solutions, so that the total differential becomes

$$
d I=I_{t} d t+I_{x} d x+I_{\dot{x}} d \dot{x}=0,
$$

where each subscript denotes partial differentiation with respect to that variable. Rewriting Eq. (2) in the form $\phi d t-d \dot{x}=0$ and adding a null term $S(t, x, \dot{x}) \dot{x} d t-S(t, x, \dot{x}) d x$ to the latter, we obtain that on the solutions the 1 -form

$$
(\phi+S \dot{x}) d t-S d x-d \dot{x}=0, \quad \phi=-(\alpha \dot{x}+\lambda x) .
$$

Hence, on the solutions, the 1-forms (6) and (7) must be proportional. Multiplying (7) by the factor $R(t, x, \dot{x})$ which acts as the integrating factors for (7), we have on the solutions that

$$
d I=R(\phi+S \dot{x}) d t-R S d x-R d \dot{x}=0 .
$$

Comparing Eq. (6) with (8) we have, on the solutions, the relations

$$
I_{t}=R(\phi+\dot{x} S), \quad I_{x}=-R S, \quad I_{\dot{x}}=-R .
$$

Then the compatibility conditions, $I_{t x}=I_{x t}, I_{t \dot{x}}=I_{\dot{x} t}, I_{x \dot{x}}=I_{\dot{x} x}$, between the Eqs. (9), provide us [22]

$$
S_{t}+\dot{x} S_{x}+\phi S_{\dot{x}}=-\phi_{x}+\phi_{\dot{x}} S+S^{2},
$$




$$
\begin{aligned}
R_{t}+\dot{x} R_{x}+\phi R_{\dot{x}} & =-\left(\phi_{\dot{x}}+S\right) R \\
R_{x}-S R_{\dot{x}}-R S_{\dot{x}} & =0 .
\end{aligned}
$$

Solving Eqs. (10)-(12) one can obtain expressions for $S$ and $R$. It may be noted that any set of special solutions $(S, R)$ is sufficient for our purpose. Once these forms are determined the integral of motion $I(t, x, \dot{x})$ can be deduced from the relation

$$
I=r_{1}-r_{2}-\int\left[R+\frac{d}{d \dot{x}}\left(r_{1}-r_{2}\right)\right] d \dot{x},
$$

where

$$
r_{1}=\int R(\phi+\dot{x} S) d t, \quad r_{2}=\int\left(R S+\frac{d}{d x} r_{1}\right) d x
$$

Equation (13) can be derived straightforwardly by integrating the Eq. (91).

As our motivation is to explore time independent integral of motion for the Eq. (2) let us choose $I_{t}=0$. In this case one can easily fix the null form $S$ from the first equation in (9) as

$$
S=\frac{-\phi}{\dot{x}}=\frac{(\alpha \dot{x}+\lambda x)}{\dot{x}} \text {. }
$$

Substituting this form of $S$ into (11) we get

$$
\dot{x} R_{x}-(\alpha \dot{x}+\lambda x) R_{\dot{x}}=-\frac{\lambda x}{\dot{x}} R
$$

Equation (15) is a first order linear partial differential equation with variable coefficients. As we noted earlier any particular solution is sufficient to construct an integral of motion (along with the function $S$ ). To seek a particular solution for $R$ one can make a suitable ansatz instead of looking for the general solution. We assume $R$ to be of the form,

$$
R=\frac{\dot{x}}{\left(A(x)+B(x) \dot{x}+C(x) \dot{x}^{2}\right)^{r}}
$$

where $A, B$ and $C$ are functions of their arguments, and $r$ is a constant which are all to be determined. We demand the above form of ansatz (16), which is very important to derive the Hamiltonian structure associated with the given equation, due to the following reason. To deduce the first integral $I$ we assume 
a rational form for $I$, that is, $I=\frac{f(x, \dot{x})}{g(x, \dot{x})}$, where $f$ and $g$ are arbitrary functions of $x$ and $\dot{x}$ and are independent of $t$, from which we get $I_{x}=\frac{f_{x} g-f g_{x}}{g^{2}}$ and $I_{\dot{x}}=$ $\frac{f_{\dot{x}} g-f g_{\dot{x}}}{g^{2}}$. From (9) one can see that $R=I_{\dot{x}}=\frac{f_{\dot{x}} g-f g_{\dot{x}}}{g^{2}}, S=\frac{I_{x}}{I_{\dot{x}}}=\frac{f_{x} g-f g_{x}}{f_{\dot{x}} g-f g_{\dot{x}}}$ and $R S=I_{x}$, so that the denominator of the function $S$ should be the numerator of the function $R$. Since the denominater of $S$ is $\dot{x}$ (vide Eq. (14)) we fixed the numerator of $R$ as $\dot{x}$. To seek a suitable function in the denominator initially one can consider an arbitrary form $R=\frac{\dot{x}}{h(x, \dot{x})}$. However, it is difficult to proceed with this choice of $h$. So let us assume that $h(x, \dot{x})$ is a function which is polynomial in $\dot{x}$. To begin with let us consider the case where $h$ is quadratic in $\dot{x}$, that is, $h=A(x)+B(x) \dot{x}+C(x) \dot{x}^{2}$. Since $R$ is in rational form while taking differentiation or integration the form of the denominator remains same but the power of the denominator decreases or increases by a unit order from that of the initial one. So instead of considering $h$ to be of the form $h=A(x)+B(x) \dot{x}+C(x) \dot{x}^{2}$, one may consider a more general form $h=\left(A(x)+B(x) \dot{x}+C(x) \dot{x}^{2}\right)^{r}$, where $r$ is a constant to be determined. The parameter $r$ plays an important role, as we see below.

Substituting (16) into (15) and solving the resultant equations, we arrive at the relation

$$
\begin{array}{r}
r\left[\dot{x}\left(A_{x}+B_{x} \dot{x}+C_{x} \dot{x}^{2}\right)-(\alpha \dot{x}+\lambda x)(B+2 C \dot{x})\right] \\
=-\alpha\left(A+B \dot{x}+C \dot{x}^{2}\right) .
\end{array}
$$

Solving Eq. (17), we can fix the forms of $A, B, C$ and $r$ and substituting them into Eq. (16) we can get the integrating factor $R$. Doing so, we find

$$
R= \begin{cases}\frac{\dot{x}}{\left(\lambda x^{2}+\alpha x \dot{x}+\dot{x}^{2}\right)}, & \alpha^{2}<4 \lambda \\ \frac{\dot{x}}{\left(\dot{x}+\frac{(r-1)}{r} \alpha x\right)^{r}}, & \alpha^{2} \geq 4 \lambda \\ \dot{x}, & \alpha=0\end{cases}
$$

where

$$
r=\frac{\alpha}{2 \lambda}\left[\alpha \pm \sqrt{\alpha^{2}-4 \lambda}\right]
$$

One can easily check the functions $S$ and $R$ given in (14) and (18), respectively, satisfy (12) also. Finally, substituting $R$ and $S$ into the form (13) for the integral we get 


$$
I=\left\{\begin{array}{c}
\frac{1}{2} \log \left(\dot{x}^{2}+\alpha x \dot{x}+\lambda x^{2}\right)+\frac{\alpha}{2 \omega} \tan ^{-1}\left[\frac{\alpha \dot{x}+2 \lambda x}{2 \omega \dot{x}}\right], \\
\alpha^{2}<4 \lambda \quad(\lambda, \alpha \neq 0) \quad \text { (underdamped) } \\
\frac{(r-1)}{(r-2)}\left(\dot{x}+\frac{\alpha}{r} x\right)\left(\dot{x}+\frac{(r-1)}{r} \alpha x\right)^{(1-r)}, \\
\alpha^{2}>4 \lambda \quad(\lambda, \alpha \neq 0) \quad \text { (overdamped) } \\
\frac{\dot{x}}{\left(\dot{x}+\frac{1}{2} \alpha x\right)}-\log \left(\dot{x}+\frac{1}{2} \alpha x\right), \quad(\text { critically damped) } \\
\alpha^{2}=4 \lambda \quad(\lambda, \alpha \neq 0) \quad \text { (pure damping) } \\
\dot{x}+\alpha x, \quad(\alpha \neq 0) \quad \text { (no damping) }
\end{array}\right.
$$

where $\omega=\frac{1}{2} \sqrt{\left(4 \lambda-\alpha^{2}\right)}$. One can easily check (using Eq. (2) ) that $\frac{d I}{d t}=0$ for each of the cases in Eq. (20). In the above, from a physical point of view we consider only the principle branches to avoid multivaluedness.

Eq. (20) demonstrates clearly that the damped linear harmonic oscillator (2) admits a time independent integral of motion for all values of the parameters $\alpha$ and $\lambda$.

Now, due to the fact that the integrals of motion (20) are time independent ones we can look for a Hamiltonian description for the respective equations of motion. In fact, in the following we do obtain the explicit time independent Hamiltonians for all the above cases.

\section{Hamiltonian description of the damped linear harmonic oscilla- tor}

Assuming the existence of a Hamiltonian

$$
I(x, \dot{x})=H(x, p)=p \dot{x}-L(x, \dot{x})
$$

where $L(x, \dot{x})$ is the Lagrangian and $p$ is the canonically conjugate momentum, we have

$$
\frac{\partial I}{\partial \dot{x}}=\frac{\partial H}{\partial \dot{x}}=\frac{\partial p}{\partial \dot{x}} \dot{x}+p-\frac{\partial L}{\partial \dot{x}}=\frac{\partial p}{\partial \dot{x}} \dot{x}
$$


From (22) we identify

$$
p=\int \frac{I_{\dot{x}}}{\dot{x}} d \dot{x}+f(x)
$$

where $f(x)$ is an arbitrary function of $x$. Equation (23) has also been derived recently by a different methodology [23]. Hereafter, without loss of generality we take $f(x)=0$. Substituting the known expression of $I$ into (23) and integrating it we can obtain the expression for the canonical momentum $p$. Substituting back the latter into the Eq. (21) and simplifying the resultant equation we arrive at the following Lagrangian

$$
L= \begin{cases}\frac{1}{2 \omega}\left(\tan ^{-1}\left[\frac{2 \dot{x}+\alpha x}{2 \omega x}\right]\left(\frac{2 \dot{x}}{x}\right)+\alpha \tan ^{-1}\left[\frac{\alpha \dot{x}+2 \lambda x}{2 \omega \dot{x}}\right]\right) & \\ -\frac{1}{2} \log \left(\dot{x}^{2}+\alpha x \dot{x}+\lambda x^{2}\right), & \alpha^{2}<4 \lambda \\ \frac{1}{(2-r)}\left(\dot{x}+\frac{(r-1)}{r} \alpha x\right)^{(2-r)}, & \alpha^{2}>4 \lambda(r \neq 0,1,2),(24) \\ \log \left(\dot{x}+\frac{1}{2} \alpha x\right), & \alpha^{2}=4 \lambda(r=2) \\ \dot{x} \log (\dot{x})-\dot{x}-\alpha x, & \lambda=0(r=1) \\ \dot{x}^{2}-\lambda x^{2}, & \alpha=0(r=0)\end{cases}
$$

and Hamiltonian

$$
H= \begin{cases}\frac{1}{2} \log \left[x^{2} \sec ^{2}(\omega x p)\right]-\frac{\alpha}{2} x p, & \alpha^{2}<4 \lambda \\ \frac{(r-1)}{(r-2)}(p)^{\frac{(r-2)}{(r-1)}}-\frac{(r-1)}{r} \alpha x p, & \alpha^{2}>4 \lambda(r \neq 0,1,2), \\ \log (p)-\frac{1}{2} \alpha x p, & \alpha^{2}=4 \lambda(r=2) \\ e^{p}+\alpha x, & \lambda=0(r=1) \\ \frac{1}{2} p^{2}+\frac{\lambda}{2} x^{2}, & \alpha=0(r=0)\end{cases}
$$

where $p$ is the canonically conjugate momentum

$$
p= \begin{cases}\frac{\tan ^{-1}\left[\frac{2 \dot{x}+\alpha x}{2 \omega x}\right]}{\omega x}, & \alpha^{2}<4 \lambda \\ \left(\dot{x}+\frac{(r-1)}{r} \alpha x\right)^{(1-r)}, & \alpha^{2} \geq 4 \lambda(r \neq 0,1) \\ \log (\dot{x}), & \lambda=0(r=1) \\ p=\dot{x}, & \alpha=0(r=0)\end{cases}
$$

respectively, for the damped harmonic oscillator (2). 
One can easily check that the canonical equations of motion for the above Hamiltonians are nothing but the equation of motion (2) of the damped linear harmonic oscillator in the appropriate parametric regimes (see below for details).

\section{Canonical Equations and Solutions}

We now write down the canonical equations of motion obtained from the above Hamiltonian expressions for each of the cases separately and obtain the corresponding solutions, as it should be, to show the correctness of our results.

\subsection{Case: $1 \alpha^{2}<4 \lambda$ :}

Now from the form of the Hamiltonian given in Eq. (25), we first derive the Hamilton's equation for the case $\alpha^{2}<4 \lambda$ :

$$
\begin{aligned}
& \dot{x}=\frac{1}{2} x(2 \omega \tan (\omega x p)-\alpha), \\
& \dot{p}=\frac{1}{2} p(\alpha-2 \omega \tan (\omega x p))-\frac{1}{x},
\end{aligned}
$$

where $\omega=\frac{1}{2} \sqrt{\left(4 \lambda-\alpha^{2}\right)}$. Rewriting Eq. (27) for $p$ and substituting it into Eq. (28), one can immediately check that the equation of motion (2) indeed follows straightforwordly from the canonical Eqs. (27)-(28). Now multiplying Eq. (28) by $x$ and (27) by $p$ and adding and integrating the resultant equation we get

$$
p=\frac{\delta-t}{x}
$$

where $\delta$ is an integration constant. Substituting the expression for $p$ into Eq. (27) we obtain the following equation for $\dot{x}$, namely,

$$
\dot{x}=\frac{1}{2} x(2 \omega \tan (\omega(\delta-t))-\alpha)
$$

which upon integration leads us to the general solution for the Eq. (2), for the choice $\alpha^{2}<4 \lambda$, of the form

$$
x(t)=C_{0} e^{-\frac{1}{2} \alpha t} \cos (\omega(t-\delta)),
$$


where $C_{0}(>0)$ is the second integration constant, as it should be for the under damped case. We also note that on substitution of the solution (31) into (20), one gets $I=H=\left(\frac{1}{2} \log C_{0}^{2}-\frac{1}{2} \alpha \delta\right)$.

4.2 Case: $2 \alpha^{2}>4 \lambda(r \neq 0,1,2$ cases $)$ :

In this case we have the following Hamilton's equation of motion

$$
\begin{aligned}
& \dot{x}=(p)^{\frac{1}{(1-r)}}-\frac{(r-1)}{r} \alpha x . \\
& \dot{p}=\frac{(r-1)}{r} \alpha p
\end{aligned}
$$

Again it is quite straightforward to check that Eqs. (32) and (33) are equivalent to (2) for $\alpha^{2}>4 \lambda(r \neq 0,1,2)$.

Integrating the above equations, we obtain the general solution as

$$
x(t)=C_{0} e^{-\frac{(r-1)}{r} \alpha t}+C_{1} \frac{r}{(r-2) \alpha} e^{-\frac{1}{r} \alpha t}
$$

where $C_{0}$ and $C_{1}(>0)$ are integration constants. Eq. (34) is the well known solution for the overdamped case. Again, we check that from (20) that $I=$ $H=\left(\frac{1-r}{r}\right) \alpha C_{0} C_{1}^{(1-r)}$.

\subsection{Case: $3 \alpha^{2}=4 \lambda(r=2)$ :}

Here the Hamilton's equations are

$$
\dot{x}=\frac{1}{p}-\frac{\alpha}{2} x, \quad \dot{p}=\frac{\alpha}{2} p .
$$

The general solution in this case is

$$
x(t)=\left(C_{0}+C_{1} t\right) e^{-\frac{1}{2} \alpha t},
$$

where $C_{0}$ and $C_{1}(>0)$ are integration constants. Here $I=H-1=1-$ $\frac{1}{2} \alpha C_{0} C_{1}^{-1}-\log C_{1}$. 


\subsection{Case: $4 \alpha \neq 0, \lambda=0(r=1)$ :}

From (25), the canonical equations for this case are

$$
\dot{x}=e^{p}, \quad \dot{p}=-\alpha,
$$

leading to the correct solution for this case,

$$
x(t)=C_{0}-\frac{1}{\alpha} e^{-\alpha\left(t-t_{0}\right)},
$$

where $C_{0}$ and $t_{0}$ are integration constants. Here $I=H=\alpha C_{0}$.

Also it may be that in each of the four cases for the associated canonical equations, the divergence of the flow function

$$
\Lambda=\frac{\partial \dot{x}}{\partial x}+\frac{\partial \dot{p}}{\partial p}=0
$$

as it should be in view of the existence of conserved Hamiltonian function.

\section{Standard Hamiltonian form}

The Hamiltonian forms given in Eq. (25) for the various parametric ranges appear rather complicated: however, they can be brought to 'standard' forms by effecting appropriate canonical transformations.

\subsection{Case: $1 \alpha^{2}<4 \lambda$ :}

With the canonical transformation $P=\frac{1}{2} \log \left(x^{2}\right)$ and $U=-x p$, the Hamiltonian for the present case, $H=\frac{1}{2} \log \left[x^{2} \sec ^{2}(\omega x p)\right]-\frac{\alpha}{2} x p$, can be transformed into

$$
H=P+\frac{1}{2} \log \left[\sec ^{2}(\omega U)\right]+\frac{\alpha}{2} U
$$




\subsection{Case: $2\left(\alpha^{2}>4 \lambda\right)$ and Case: $3\left(\alpha^{2}=4 \lambda\right)$ :}

For these two cases, the Hamiltonian functions given in Eq. (25) get transformed through the canonical transformation $x=-P e^{-U}$ and $p=e^{U}$ as

$$
H= \begin{cases}P+\frac{r}{(r-2) \alpha} e^{\frac{(r-2)}{(r-1)} U}, & \alpha^{2}>4 \lambda(r \neq 0,1,2) \\ P+\frac{2}{\alpha} U, & \alpha^{2}=4 \lambda .\end{cases}
$$

5.3 Case: $4 \alpha \neq 0, \lambda=0(r=1)$ :

In this case, the Hamiltonian $H=e^{p}+\alpha x$ transformed into the form

$$
H=P+e^{-\alpha U}
$$

through the canonical transformation $x=\frac{P}{\alpha}$ and $p=-\alpha U$.

Since the canonical variables are now separated, the new Hamiltonians can be utilized for applications including the quantization.

Before proceeding further, we wish to point out that an explicit time independent integral for the damped harmonic oscillator (2) was given by López and López $[23,24]$. However, no explicit Hamiltonian could be associated with it. We trace the reason for this in Appendix-A.

\section{Quantum description}

Having obtained appropriate Hamiltonian formalism, we now proceed to identify a possible quantum description of the damped linear harmonic oscillator. For this purpose, we first rewrite the classical Hamiltonian in a symmetrized form so as to ensure hermiticity when the dynamical variables are represented by linear operators acting on a quantum Hilbert space. Thus we write 


$$
H= \begin{cases}\frac{1}{2} \log \left[x^{2} \sec ^{2}\left(\frac{\omega}{2}(x p+p x)\right)\right]-\frac{\alpha}{4}(x p+p x), & \alpha^{2}<4 \lambda \\ \frac{(r-1)}{(r-2)}(p)^{\frac{(r-2)}{(r-1)}}-\frac{(r-1)}{2 r} \alpha(x p+p x), & \alpha^{2}>4 \lambda(r \neq 0,1,2), \\ \log (p)-\frac{\alpha}{4}(x p+p x), & \alpha^{2}=4 \lambda(r=2) \\ e^{p}+\alpha x, & \lambda=0(r=1) \\ \frac{1}{2} p^{2}+\frac{\lambda}{2} x^{2}, & \alpha=0(r=0) .\end{cases}
$$

In the above $x$ and $p$ are now considered as linear operators satisfying the cannonical commutation rule $(\mathrm{CR})$

$$
[x, p]=i \hbar
$$

Obviously, one can look for a coordinate representation in which $x$ is treated as a variable and $p$ as the operator $-i \hbar \frac{\partial}{\partial x}$ so that the CR (44) is satisfied or in a momentum representation one may treat $p$ as a c-number and $x$ as the operator $x=i \hbar \frac{\partial}{\partial p}$ satisfying (44). For the present problem we choose a momentum representation and write the time-dependent Schrödinger equation as

$$
i \hbar \frac{\partial \Psi(p, t)}{\partial t}=H \Psi(p, t)
$$

Next we look for the stationary states

$$
\Psi(p, t)=\psi(p) e^{-\frac{i \mathcal{E} t}{\hbar}}
$$

so that (45) reduces to the time-independent Schrödinger equation in the momentum space:

$$
H \psi(p)=\mathcal{E} \psi(p)
$$

Here $\mathcal{E}$ is identified as the eigenvalue corresponding to the Hamiltonian operator $H$. In the following we first consider the pure damped case, that is, $\lambda=0$ and then the remaining cases (except the case $\alpha^{2}<4 \lambda$ ). We wish to note one can consider the quantization of the transformed Hamiltonians (40)-(42) also which is discussed in Appendix B. 


\subsection{Case: $1 \lambda=0$ :}

In the case $\lambda=0$, the Hamiltonian $H=e^{p}+\alpha x$ is quantized so that the time independent Schrödinger equation in the momentum space becomes

$$
H \psi=\mathcal{E} \psi \Rightarrow\left(i \alpha \hbar \frac{d}{d p}+e^{p}\right) \psi=\mathcal{E} \psi
$$

Solving the above spectral problem for any real value of $\mathcal{E},-\infty<\mathcal{E}<\infty$, we obtain the wavefunction in the form

$$
\psi(p)=C_{1} \exp \left[i\left(e^{p}-\frac{p \mathcal{E}}{\alpha \hbar}\right)\right]
$$

where $C_{1}$ is the normalization constant. From equations (46) and (49) we get the plane wave like solution

$$
\Psi(p, t)=C_{1} \exp \left[-\frac{i \mathcal{E} t}{\hbar}+i\left(\frac{e^{p}-p \mathcal{E}}{\alpha \hbar}\right)\right], \quad-\infty<\mathcal{E}<\infty
$$

as the final solution of (47).

\subsection{Case: $2 \alpha^{2}=4 \lambda$ :}

For the case $\alpha^{2}=4 \lambda$, the eigen value equation becomes

$$
H \psi=\mathcal{E} \psi \Rightarrow\left(\log (p)-i \frac{\hbar \alpha}{4}\left(1+2 p \frac{d}{d p}\right)\right) \psi=\mathcal{E} \psi
$$

Introducing the transformation of the form $\hat{\psi}=\psi e^{i \frac{1}{\hbar \alpha}(\log (p))^{2}}$ into the above equation we get

$$
p \frac{d \hat{\psi}}{d p}=\left[-\frac{1}{2}+i \frac{2 \mathcal{E}}{\hbar \alpha}\right] \hat{\psi}, \quad-\infty<\mathcal{E}<\infty .
$$

The above eigenvalue equation is exactly of the same form as that of the eigenvalue equation in the coordinate space for the toy model for quantum damping introduced by Chruscinski [25], see Eq. (6.2), in a rigged Hilbert space.

Consequently the generalized eigenfunctions can be given as 


$$
\hat{\psi}(p):=\frac{1}{\sqrt{-\pi \alpha \hbar}} p_{ \pm}^{i \frac{2 \mathcal{E}}{\alpha \hbar}-\frac{1}{2}}
$$

where following Ref. [25] the tempered-distributions are defined as

$$
\begin{aligned}
& p_{+}^{\lambda}:= \begin{cases}p^{\lambda}, & p \geq 0 \\
0, & p<0\end{cases} \\
& p_{-}^{\lambda}:= \begin{cases}0, & p \geq 0, \\
|p|^{\lambda}, & p<0,\end{cases}
\end{aligned}
$$

with $\lambda \in \mathbf{C}$. The resultant wavefunction becomes

$$
\psi(p)=\frac{1}{\sqrt{-\pi \alpha \hbar}} p_{ \pm}\left(i \frac{2 \mathcal{E}}{\alpha \hbar}-\frac{1}{2}\right) e^{-i\left(\frac{(\log (p))^{2}}{\hbar \alpha}\right)} .
$$

In Ref. [25], Chruscinski has shown that the complex eigenvalues of the underlying Hamiltonian correspond to the poles of energy eigenvectors when continued to the complex eigenvalue plane and that the corresponding generalized eigenvectors may be interpreted as resonant states and these resonant states are responsible for the irreversible quantum dynamics. Consequently, similar arguments can hold good here also. Detailed description will be given elsewhere.

\subsection{Case: $3 \alpha^{2}>4 \lambda$ :}

In this case we have the following eigen value equation to be solved,

$$
H \psi=\mathcal{E} \psi \Rightarrow\left(\frac{(r-1)}{(r-2)}(p)^{\frac{(r-2)}{(r-1)}}-i \frac{(r-1)}{2 r} \alpha \hbar\left(1+2 p \frac{d}{d p}\right)\right) \psi=\mathcal{E} \psi
$$

As we did earlier let us introduce the transformation

$$
\hat{\psi}=\psi e^{i\left(\frac{r(r-1)}{\alpha \hbar(r-2)^{2}} p^{\frac{r-2}{r-1}}\right)},
$$


into equation (57) and we obtain the eigenvalue equation

$$
p \frac{d \hat{\psi}}{d p}=-\left[i \frac{r \mathcal{E}}{(1-r) \alpha \hbar}+\frac{1}{2}\right] \hat{\psi}, \quad-\infty<\mathcal{E}<\infty
$$

Correspondingly, again following Chruscinski [25] the generalized eigenfunctions can be given as

$$
\hat{\psi}(p):=\sqrt{\frac{r}{2(1-r) \pi \alpha \hbar}} p_{ \pm}-\left(i \frac{r \mathcal{E}}{(1-r) \alpha \hbar}+\frac{1}{2}\right) .
$$

Then the wavefunction becomes

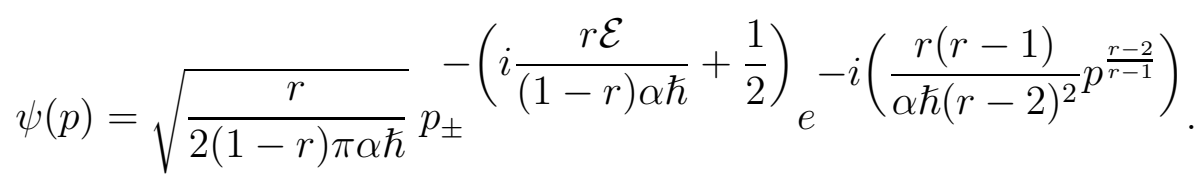

Again the properties of the generalized eigenfunctions discussed in Ref. [25] hold good here also.

Finally for the case $\alpha^{2}<4 \lambda$ in Eq. (43), it is not yet clear how to solve the linear eigenvalue problem. However, another possible approach is to make use of the canonically transformed Hamiltonian of the form (40) as indicated in Appendix B.

\section{Conclusions}

In this paper, we have identified explicit time independent first integrals for the damped linear harmonic oscillator valid in different parameter regimes using the modified Prelle- Singer approach. We have constructed the appropriate Lagrangians and explicit Hamiltonians from the time independent first integrals and transformed the corresponding Hamiltonian forms to standard Hamiltonian forms using suitable canonical transformations. We have also indicated that a quantized description can be developed in the momentum representation using the Hamiltonian forms and map onto known quantum mechanical toy models of damped systems. We expect that further analysis of the quantum case can reveal much information on the quantum damped oscillator. 


\section{Acknowledgments}

The authors wish to thank Professor R. Simon, Institute of Mathematical Sciences, Chennai for a useful discussion. The work of VKC is supported by CSIR in the form of a CSIR Senior Research Fellowship. The work of ML forms part of a Department of Science and Technology, Government of India sponsored research project and is supported by a Department of Atomic Energy Raja Ramanna Fellowship.

\section{A Appendix}

A set of first integrals for the damped harmonic oscillator (2) was reported in Refs. [23, 24] in the form

$$
K=\frac{1}{2}\left(\dot{x}^{2}+\alpha x \dot{x}+\lambda x^{2}\right) e^{-\alpha G}
$$

where

$$
G= \begin{cases}\frac{2}{\sqrt{4 \lambda-\alpha^{2}}} \tan ^{-1}\left[\frac{2 \dot{x}+\alpha x}{\sqrt{4 \lambda-\alpha^{2}} x}\right], & \alpha^{2}<4 \lambda \text { (underdamped) } \\ \frac{1}{\sqrt{\alpha^{2}-4 \lambda}} \log \left(\frac{\left(\alpha-\sqrt{\alpha^{2}-4 \lambda}\right) x+2 \dot{x}}{\left(\alpha+\sqrt{\alpha^{2}-4 \lambda}\right) x+2 \dot{x}}\right), & \alpha^{2}>4 \lambda \text { (overdamped) } \\ \frac{4 \dot{x}}{2 \alpha \dot{x}+\alpha^{2} x}, & \alpha^{2}=4 \lambda \text { (critically damped) }\end{cases}
$$

Note that in Refs. $[23,24]$ the value of $G$ for the critically damped case is given as $G=\frac{1}{\omega_{\alpha}+\frac{v}{x}}$ but the correct form is $G=\frac{1}{\omega_{\alpha} \frac{x}{v}\left(\omega_{\alpha}+\frac{v}{x}\right)}$.

All the first integrals given in Eq. A.1 are related to our first integrals (Eq. (20)-underdamped, overdamped, critically damped cases) in the following manner (after using suitable transformation formulas)

$$
I= \begin{cases}\frac{1}{2} \log (2 K)-\frac{\alpha}{\sqrt{4 \lambda-\alpha^{2}}} \tan ^{-1}\left[\frac{\sqrt{4 \lambda-\alpha^{2}}}{\alpha}\right], & \alpha^{2}<4 \lambda \text { (underdamped) } \\ \frac{(r-2)}{(r-1)}(2 K)^{\frac{(2-r)}{2}}, & \alpha^{2}>4 \lambda \text { (overdamped) } \\ -\frac{1}{2} \log (2 K), & \alpha^{2}=4 \lambda \text { (critically damped) }\end{cases}
$$

where $r$ is the quantity defined in Eq. (19)).

In Refs. [23,24], the authors have found the Lagrangian and an implicit Hamiltonian only for underdamped case $\left(\alpha^{2}<4 \lambda\right)$ and concluded that the dissipative 
harmonic oscillator has nonexplicit Hamiltonian formulation. For the other cases it was stated that it is not possible to get a close expression for the Lagrangian. However in the present work we have found the Lagrangian and explicit Hamiltonian for all the three cases.

The reason for not getting the Lagrangian and generalized linear momentum from the first integral (A.1) reported in Refs. [23, 24] is as follows:

For example, the first integral $I$ given in Eq. (201) for the overdamped case is related to the first integral $K$ reported in Refs. [23, 24] through the relation $I=\frac{(r-2)}{(r-1)}(2 K)^{\frac{(2-r)}{2}}$. Our first integral $I$ for the overdamped case unambiguously leads to the generalized linear momentum, Lagrangian and explicit Hamiltonian from Eqs. (21) and (23) whereas integral $K$ does not. The reason for the above is not difficult to understand. For example, let us consider the first integral $I=\dot{x}^{2}+\omega^{2} x^{2}$ for the linear harmonic oscillator which gives the conjugate momentum $p=\dot{x}$ and Lagrangian $L=\dot{x}^{2}-\omega^{2} x^{2}$ (vide Eqs. (21) and (23)). However, $\hat{I}=\left(\dot{x}^{2}+\omega^{2} x^{2}\right)^{n}$, where $n$ is some arbitrary parameter, is also a first integral for SHO. By substituting this first integral $\hat{I}$ into the expressions (21) and (23), the linear momentum and Lagrangian cannot be obtained in terms of any closed form. In order to find the Lagrangian and Hamiltonian from the known first integral, one has to take appropriate form of the first integral. Our PS method gives directly the correct form of first integral needed to find the Lagrangian and Hamiltonian of the system.

\section{B Quantum description in standard Hamiltonian form}

Let us consider the Eqs. (40) - (42), which are all of the form

$$
H=P+g(U),
$$

where

$$
g(U)= \begin{cases}\frac{1}{2} \log \left[\sec ^{2}(\omega U)\right]+\frac{\alpha}{2} U, & \alpha^{2}>4 \lambda \\ \frac{r}{(r-2) \alpha} e^{\frac{(r-2)}{(r-1)}}, & \alpha^{2}>4 \lambda(r \neq 0,1,2), \\ \frac{2}{\alpha} U, & \alpha^{2}=4 \lambda \\ e^{-\alpha U}, & \lambda=0 .\end{cases}
$$

The Hamiltonian (B.1) is now quantized in the new coordinate space, so that here $P=-i \hbar \frac{\partial}{\partial U}$ and the corresponding time independent Schródinger equation becomes 


$$
H \psi=E \psi \Rightarrow\left(-i \hbar \frac{d}{d U}+g(U)\right) \psi=\mathcal{E} \psi
$$

Introducing the transformation $\hat{\psi}=\psi e^{i \frac{\int g(U) d U}{\hbar}}$ into $(\underline{B .3})$ we get

$$
\frac{d \hat{\psi}}{d U}=i \frac{\mathcal{E}}{\hbar} \hat{\psi}
$$

Solving the above one can get the form of $\hat{\psi}$, namely,

$$
\hat{\psi}(U)=C_{1} e^{i \frac{\mathcal{E} U}{\hbar}}
$$

Then the wavefunction $\psi$ becomes

$$
\psi(U)=C_{1} e^{i\left(\frac{\mathcal{E} U-\int g(U) d U}{\hbar}\right)} .
$$

Here

$$
\int g(U) d U= \begin{cases}\frac{1}{2}\left(U \log \left(\sec ^{2}(\omega U)\right)+2 U \log \left(1+e^{2 i \omega U}\right)\right. & \\ \left.\quad+\frac{\alpha}{4} U^{2}-i\left(\omega U^{2}+\frac{1}{\omega} L i_{2}\left(-e^{2 i \omega U}\right)\right)\right), & \alpha^{2}<4 \lambda \\ \frac{(r-1) r}{\alpha(r-2)^{2}} e^{\frac{(r-2)}{(r-1)} U}, & \alpha^{2}>4 \lambda \\ \frac{1}{\alpha} U^{2}, & \alpha^{2}=4 \lambda \\ -\frac{1}{\alpha} e^{-\alpha U}, & \lambda=0\end{cases}
$$

where $L i_{n}(z)$ is polylogarithm function [26]. In order to relate this form of the wavefunctions in terms of the original variable one has to make use of the appropriate canonical transformations.

\section{References}

[1] V. K. Chandrasekar, M. Senthilvelan, M. Lakshmanan, Phys. Rev. E 72 (2005) 066203.

[2] V. K. Chandrasekar, S. N. Pandey, M. Senthilvelan and M. Lakshmanan, J. Math. Phys. 47 (2006) 023508.

[3] H. Goldstein, Classical Mechanics, Addison Wesley, Reading, MA, 1950.

[4] J. R. Ray, Am. J. Phys. 47 (1979) 626-629. 
[5] F. Riewe, Phys. Rev. E 53 (1996) 1890.

[6] H. Dekker, Phys. Rev. A 16 (1977) 2126.

[7] H. Dekker, Phys. Rep. 80 (1981) 1.

[8] H. Gzyl, Phys. Rev. A 27 (1983) 2297.

[9] E. G. Harris, Phys. Rev. A 42 (1990) 3685.

[10] S. Srivastava, Vishwamittar, I. S. Minhas, J. Math. Phys. 32 (1991) 1510.

[11] E. G. Harris, Phys. Rev. A 48 (1993) 995.

[12] R. Egger, H. Grabert, U. Weiss, Phys. Rev. E 55 (1997) R3809.

[13] R. Karrlein and H. Grabert, Phys. Rev. E 55 (1997) 153.

[14] M. Blasone, P. Jizba, G. Vitiello, Phys. Lett. A 287 (2001) 205.

[15] C. I. Um, K. H. Yeon, T. F. George, Phys. Rep. 362 (2002) 63.

[16] D. C. Latimer, J. Phys. A 35 (2002) 5591; D. C. Latimer, J. Phys. A 38 (2005) 2021.

[17] A. Pereverzev, Phys. Rev. E 68 (2003) 026111.

[18] J. R. Choi, Rep. Math. Phys. 52 (2003) 312.

[19] D. Chruscinski, J. Jurkowski, Ann. Phys. 321 (2006) 854; D. Chruscinski, Ann. Phys. 321 (2006) 840.

[20] G. Dito, F. J. Turrubiates, Phys. Lett. A 352 (2006) 309.

[21] V. K. Chandrasekar, M. Senthilvelan, M. Lakshmanan, Proc. R. Soc. London 461 (2005) 2451; 462 (2006) 1831.

[22] L. G. S. Duarte, S. E. S. Duarte, A. C. P. da Mota, J. E. F. Skea, J. Phys. A 34 (2001) 3015.

[23] G. López, Ann. Phys. 251 (1996) 372.

[24] G. López, P. López, Int. J. Theor. Phys. 45 (2006) 734.

[25] D. Chruscinski, J. Math. Phys. 44 (2003) 3718; D. Chruscinski, J. Math. Phys. 45 (2004) 841.

[26] M. Abramowitz and I. A. Stegun, eds. Handbook of Mathematical Functions with Formulas, Graphs, and Mathematical Tables, Dover, New York, (1972). 